\title{
COHYPONORMAL OPERATORS WITH THE SINGLE VALUED EXTENSION PROPERTY
}

\section{RIDGLEY LANGE and SHENGWANG WANG}

Department of Mathematics

Central Michigan University

Mt. Pleasant, Michigan 48859 U.S.A.

(Received September 26, 1985)

ABSTRACT. It is proved that in order to find a nontrivial hyperinvariant subspace for a cohyponormal operator it suffices to make the further assumption that the operator have the single-valued extension property.

KEY WORDS AND PHRASES. Cohyponormal, single valued extension property 1980 AMS SUBJECT CLASSIFICATION CODE. 47A15, 47B20, $47 B 40$.

\section{INTRODUCTION.}

Let $T$ be a cohyponormal operator on the complex Hilbert space $X$. This means that $T^{\star}$ is hyponormal, so $\|T x\|<\left\|T^{\star} x\right\|$ for each vector $x$. The aim of this paper is to reduce the problem of existence of a nontrivial invariant (or hyperinvariant) subspace for $T$ to the case where $T$ has the single-valued extension property and is the compact perturbation of a normal operator (see $\S 3$ ). In $\S 2$ we record some consequences of a recent theorem of Putinar [1] that every hyponormal operator is "subscalar". The last section gives some further invariant subspace results for this reduced case.

We mention a recent paper of S. Brown [2] in which the author shows that a hyponormal operator having spectrum with nonempty interior has invariant (but not necessarily hyperinvariant) subspaces.

2. HYPONORMAL OPERATORS.

M. Putinar [1] has recently proved that every hyponormal is "subscalar" in the sense of [3, Def. 1.2, p. 94]. The precise statement is as follows.

THEOREM 1. Let $T$ be hyponormal on $X$. Then $T$ has an extension $S$ on a Hilbert space $Y$ containing $X$ such that $S$ is a generalized scalar operator of order 2 . In particular, $S$ in decomposable [3, p. 67].

Every decomposable operator has property $(B)$ [4], introduced by Bishop [5]; $T$ has property $(B)$ if, for each sequence of analytic functions $f_{n}: D \rightarrow X$ such that $(\lambda-T) f_{n}(\lambda)+0$ uniformly on compact sets, $f_{n} \rightarrow 0$ uniformly on compact sets. Since it is clear that restrictions inherit $(\beta)$, the following is immediate.

COROLLARY 1. Every hyponormal operator has property $(\beta)$. 
COROLLARY 2. If $T$ is hyponormal and has a nonzero invariant subspace $M$ such that $\sigma(T \mid M) \subset \sigma(T)$ is proper, then $T$ has a nontrivial hyperinvariant subspace.

PROOF. It follows from Corollary 1 that the spectral manifold $N=X_{T}(O(T \mid M))$ is closed, hyperinvariant for $T$, and contains $M$. Since $\sigma(T \mid M) \neq \sigma(T)$, $N$ is nontrivial. 3. COHYPONORMAL OPERATORS.

THEOREM 2. Let $T$ be cohyponormal on $X$. Then $T$ is decomposable iff it has property $(\beta)$.

PRO0F. If $T$ has property $(\beta)$, then it is decomposable by Corollary 1 and [6]. The converse was noted in $\$ 1$.

REMARK 1. The left shift on $\ell^{2}(N)$ is cohyponormal but it does not have property $(B)$ since it is not decomposable. On the other hand, there do exist nonnormal decomposable cohyponormal operators satisfying $(\beta)$. Radjabalipour [7] has proved the existence of nonnormal decomposable subnormal operators; adjoints of such operators are cohyponormal and satisfy the conclusion of Theorem 2.

COROLLARY 3. Let $T$ be a cohyponormal operator which is not a scalar multiple of the identity on $X$. If $T$ satisfies $(\beta)$, then it has a nontrivial hyperinvariant subspace.

PR00F. If $\sigma(T)$ is the singleton $\{\lambda\}$, then $(\lambda-T) \star$ is a quasinilpotent hyponormal opertor. Hence $\lambda-T=0$, contradiction. Now $T$ is decomposable by Theorem 2 , and since $\sigma(T)$ has at least two points, $T$ has a nontrivial, hyperinvariant spectral manifold $X_{T}(F)$.

COROLLARY 4. Let $T$ be cohyponormal and have property ( $\beta)$. If for each hyperinvariant subspace $M$ the restriction $T / M$ is cohyponormal, then $T$ is strongly decomposable.

PRO0F. Let $M=X_{T}(F)$ for $F$ closed. Since $T \mid M$ is cohyponormal by hypothesis and inherits $(B)$, then $T / M$ is decomposable by Theorem 2. This is just the definition that $T$ is strongly decomposable [8].

REMARK 2. Since the subnormal operators mentioned in Remark 1 in fact have spectral distributions, their adjoints are strongly decomposable cohyponormal operators but nonnormal (see [3, p. 65 and p. 81]).

In general it is not known if $(\beta)$ is preserved under the Dunford functional calculus, but it is in the case of cohyponormal operators (Corollary 5). We note that $(\beta)$ is also preserved in this way for hyponormal operators as well. For let $T$ be hyponormal on $X$ and let $f$ be analytic on some region containing $\sigma(T)$. If $S$ is the scalar extension of Theorem 1, then $\sigma(S) \subset \sigma(T)[1$, Cor 2.6] so $f(S)$ is defined and decomposable [3, p. 37] and $f(T)=f(S) \mid X$. Thus $f(T)$ satisfies $(\beta)$.

COROLLARY 5. Let $T$ be cohyponormal and let $f$ be analytic on $\sigma(T)$. Then $f(T)$ satisfies ( $\beta$ ) if $T$ does. Conversely, if $f$ is nonconstant on each component of its domain, then $T$ satisfies $(\beta)$ if $f(T)$ does.

PR00F. If $T$ has property $(\beta)$, then it is decomposable by Theorem 2 . Hence $f(T)$ is also decomposable and so satisfies $(\beta)[4]$. For the converse, note that $f$ (the conjugate of $f$ ) is analytic on $\sigma\left(T^{\star}\right)$. Thus if $S$ is the scalar extension of $T^{*}$ (Theorem 1), then $f(S)$ is decomposable and $f\left(T^{*}\right)$ has property $(B)$. By [6] $f(T)$ is decomposable, so by [8] $T$ is decomposable and thus satisfies ( $\beta$ ). 
REMARK 3. Corollary 5 may fail if $f$ is anywhere constant. If $T$ is the left shift and $f \equiv 1$, then $f(T)=I$ has property $(B)$ but $T$ does not.

Property ( $B$ ) implies the (strictly) weaker single-valued extension property (SVEP) which has had a role in the theory of spectral decomposition (see [3, p. 1]). We shall see below that it may also be significant in the invariant subspace problem for hyponormal operators. (An example in [3, p. 25] shows SVEP does not imply (B). The manifold $X_{T}(\{0\})$ constructed there is not closed, so (B) fails [4]).

PROPOSITION 1. Let $T$ be cohyponormal satisfying SVEP. If $S$ is the scalar extension of $T^{\star}$, then $\sigma(S)=\sigma\left(T^{\star}\right)$.

PRO0F. Since we know $\sigma(S) \subset \sigma\left(T^{\star}\right)\left[1\right.$, Cor. 2.6], suppose $\lambda \varepsilon \sigma\left(T^{\star}\right)-\sigma(S)$. Then $\lambda-S$, and hence $\lambda-T^{\star}$, is bounded below. Since $\lambda-T^{\star}$ is hyponormal, it is semi-Fredholm with index at most zero. If ind $\left(\lambda-T^{\star}\right)<0$, then ind $\left(\mu-T^{\star}\right)<0$ for $\mu$ sufficiently near $\lambda$. Hence $\bar{\mu}$ is an eigenvalue of $T$ for all such $\mu$. Moreover, $\bar{\lambda}-T$ is also semi-Fredholm, so by [9, Th. 9] $T$ does not have SVEP, contradiction. Hence $\sigma(S)=\sigma\left(T^{\star}\right)$.

As corollaries to the previous proof we have the following:

COROLLARY 6. If $T$ is cohyponromal and has SVEP, then $T$ is biquasitriangular.

PROOF. For $\lambda$ in the essential resolvent set of $T^{\star}$ the previous proof implies ind $\left(\lambda-T^{*}\right)=0$. By [10] $T^{\star}$, and so $T$, is biquasitriangular.

COROLLARY 7. Let $T$ be hyponormal with scalar extension $S$. If $\sigma(S) \neq \sigma(T)$, then $T$ has a nontrivial hyperinvariant subspace.

PROOF. Since $\sigma(S)$ is proper in $\sigma(T)$, the proof of Proposition 1 shows that $T^{\star}$ does not have the SVEP. Hence $T^{\star}$ has an eigenvector, so $T$ has a nontrivial hyperinvariant subspace.

COROLLARY 8. Let $T$ be a cohyponormal operator which is not a scalar multiple of the identity. Then $T$ has a nontrivial hyperinvariant subspace if $T$ has property $(\beta)$ or $T$ does not have SVEP.

PROOF. If $T$ satisfies $(\beta)$, use Corollary 3. In the other case $T$ has at least one eigenvector.

By Corollary 8 the hyperinvariant subspace problem for a hyponormal operator is reduced to the case where its adjoint has SVEP but not property $(B)$. This leads to the related question: if $T$ is cohyponomal does SVEP imply $(\beta)$ ? An affirmative answer to the last question would, of course, resolve the former one. On the other hand, if our interest is merely invariant subspaces, we have:

THEOREM 3. If $T$ is cohyponomal, then either $T$ has a nontrivial invariant subspace or $T$ is a compact perturbation of a normal operator.

PRO0F. By Corollary 8 we may suppose that $T$ has SVEP. If $T^{\star}$ has a cyclic vector, we may suppose it is rationally cyclic. By [11, Th. 4] T*T - TT* is in the trace class. By Proposition 1 and [12, Th. 11.11] $T=N+K$ where $N$ is normal and $K$ is compact.

Our results now indicate that to solve the hyperinvariant subspace problem for cohyponormal $T$ we may make the following additional assumptions:

(a) $T$ is the compact perturbation of a normal operator and $\sigma\left(T^{\star}\right)=\sigma(S)$ where $S$ is the scalar extension of $T^{*}$. 
(b) T satisfies SVEP but not ( $\beta$ ).

(c) for at least one open disc $D$ the manifold $x_{T}(C-D)$ is not closed but dense in $X$; otherwise $T$ has already a hyperinvariant (nontrivial) subspace.

4. INVARIANT SUBSPACES.

In this section we derive some invariant subspace results for hyponorinal operators in light of the reduction given in the last section. Given an operator $T$, we write $R(T)$ for the uniform closure of rational functions $f(T)$ where $f$ has poles off $\sigma(T)$. Let $\underline{K}_{1}$ be the set of compact operators on $X$ of norm 1 . Finally, recall that $\sigma(T)$ is c-spectral for $T[13]$ if there exists $c>0$ such that

$$
\|f(T)\|<c\|f\| \sigma(T)
$$

where $f(T) \varepsilon R(T)$ and the last norm is the sup-norm of $f$ on $\sigma(T)$.

COROLLARY 9. If $T$ is hyponormal, then it has a nontrivial invariant subspace in each of the following cases.

(i) distance from $R(T)$ to $\underline{K}_{1}$ is positive;

(ii) the image of $R(T)$ is closed in the Calkin algebra;

(iii) the image of $R(T)$ is closed under the Gelfand transform.

PRO0F. We may suppose (a) holds for $T^{\star}$. In this case, by [14, Th. 1] (i) (iii) are equivalent to $\sigma(T)$ being c-spectral for $T$. So $T$ has a proper invariant subspace by [13, Th. 2].

COROLLARY 10. Let $T$ be hyponormal with scalar extension $S$. If $\sigma(S)$ is $c-$ spectral for $S$, then $T$ has a nontrivial invariant subspace $M$. Moreover, $T$ has a nontrivial hyperinvariant subspace or T/M itself has a nontrivial invariant subspace.

PROOF. Since $\sigma(S) \subset \sigma(T)$ it is easy to check that $\sigma(T)$ is c-spectral for $T$, hence $T$ has a nontrivial invariant subspace $M$ by [13, Th. 2].

To prove the second statement we consider several cases for $\sigma(T / M)$. I. $\sigma(T \mid M) \subset \sigma(T)$ is proper: use Corollary 2. II. $\sigma(T \mid M)$ contains a bounded component of $\rho(T)$ : let $\lambda \varepsilon \rho(T)-\sigma(T \mid M)$. It follows that $\lambda$ is an eigenvalue of $(T \mid M)$ * so $T \mid M$ has a proper (hyper) - invariant subspace. III. $\sigma(T) \subset \sigma(T / M)$ : this implies $\sigma(T \mid M)$ is C-spectral for T/M, so T/M has a proper invariant subspace by [13].

LEMMA 1. Let $N$ be normal and suppose for an operator $T$ that $(\lambda-T) X \subset(\lambda-N) X$ for all $\lambda \in F$ where $F$ is some closed set. Then $X_{N}(F)$ is T-invariant.

PR00F. Since $N$ is normal, by $[15] X_{N}(F)=n_{\lambda \notin F}(\lambda-N) X$. Hence for $f \in X_{N}(F)$ we have $f \varepsilon(\lambda-N) X$ for $\lambda \& F$. Let $\mu \varepsilon F$. Then $(\mu-T) f \varepsilon(\mu-N) X$, so for some $g \in X$

$$
T f=\mu f+(\mu-N) g \varepsilon(\mu-N) X
$$

Thus Tf $\varepsilon X_{N}(F)$ and lemma is proved.

PROPOSITION 2. Let $T$ be hyponormal such that $T=N+K$ where $N$ is normal and $K$ is the rank-one operator defined by $K f=(u, f) v$ for all $f E x$, and fixed $u, v E x$. Let $F \subset \sigma(N)$ be proper, closed such that $v \in(\lambda-N) X$, all $\lambda$ \&F. Then $T$ has a nontrivial invariant subspace. Furthermore, if $N$ has no point spectrum, then $T$ has a nontrivial hyperinvariant subspace.

PROOF. Since the conclusion is clear if $T$ has an eigenvalue, we suppose $T$ has empty point spectrum. Now $K f$ is a scalar multiple of $v$ for all $f$, so if we let $g \in X, \lambda \varepsilon F$, 


$$
(\lambda-T) g=(\lambda-N) g+\alpha v \varepsilon(\lambda-N) X . \quad(\alpha \text { complex }) .
$$

Hence $(\lambda-T) X \subset(\lambda-N) X$ for all $\lambda \notin F$. By the lemma, $X_{N}(F)$ is T-invariant. Since $0 \neq v \varepsilon X_{N}(F)$ and $F$ is proper in $\sigma(N), X_{N}(F)$ is nontrivial.

Put $U=X_{N}(F)$. Then $T|U=N| U+K \mid U$, so by Weyl's Theorem $\sigma(T \mid U) \subset \sigma(N \mid U) \subset F \subset \sigma(N)$. If we suppose $N$ has no eigenvalues, then $\sigma(N)=\sigma(T)$ by Weyl's Theorem, and $\sigma(T \mid U)$ is properly contained in $\sigma(T)$. By Corollary $2 T$ has a proper hyperinvariant subspace.

\section{REFERENCES}

1. PUTINAR, M., Hyponormal operators are subscalar, J. Operator Theory $12(1984), 385-$ 395.

2. BROWN, S. W., Hyponomal operators with thick spectra have invariant subspaces, preprint.

3. COLOJOARA, I., FOIAS, C., Theory of generalized spectral operators, Gordon and Breach, New York, 1968.

4. FOIAŞ, C., On the maximal spectral spaces of a decomposable operator, Rev. Roum. Math. Pures Appl. 15(1970), 1599-1606.

5. BISHOP, E., A duality theorem for an arbitrary operator, Pacific J. Math. 9 (1959), 379-397.

6. LANGE, R., A purely analytic criterion for a decomposable operator, Glasgow Math. J. 21 (1980), 69-70.

7. RADJABALIPOUR, M., Some decomposable subnormal operators, Rev. Roum. Math. Pures Appl. 22(1977), 341-345.

8. APOSTOL, C., Roots of decomposable operator valued analytic functions, Rev. Roum. Math. Pures Appl. 13(1968), 433-438.

9. FINCH, J., The single valued extension property on Bauach space, Pacific J. Math. 25 (1975), 61-69.

10. APOSTOL, C., FOIAS,, C., VOICULESCU, D., Some results on nonquasitriangular operators IV, Rev. Roum. Math. Pures Appl. 18 1973$), 387-514$.

11. BERGER, C. A., SHAW, B. I., Intertwining, analytic structure, and the trace norm estimate, In Proc. Conf. Operator Theory, Lecture Notes in Math. 345, Springer, New York, 1973.

12. BROWN, L. G., DOUGLAS, R. G., FILLMORE, P. A., Unitary equivalence module the compact operators and extensions of $C^{\star}$-algebras, In Proc. Conf. Operator Theory, Lecture Notes in Math. 345, Springer, New York, 1973.

13. STAMPFLI, J. G., An extension of Scott Brown's theorem: K-spectral sets, J. Operator Theory $3(1980), 3-21$.

14. LANGE, R., Essentially subnormal operators and K-spectral sets, Proc. Amer. Math. Soc. 88(1983), 449-453.

15. PTÁK, V., VRBOVÁ, P., On the spectral function of a normal operator, Czechoslovak Math. J. 23(98), 615-616. 


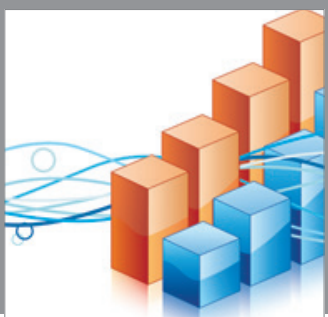

Advances in

Operations Research

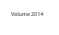

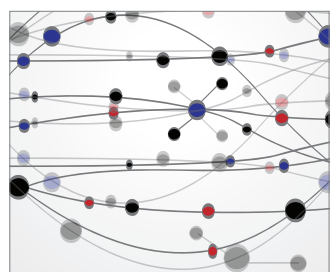

\section{The Scientific} World Journal
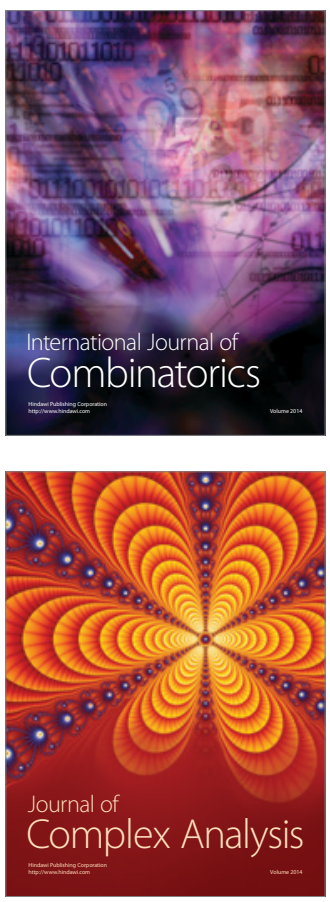

International Journal of

Mathematics and

Mathematical

Sciences
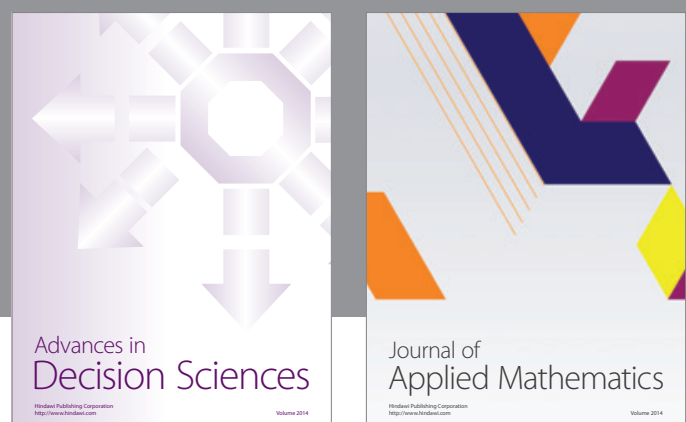

Journal of

Applied Mathematics
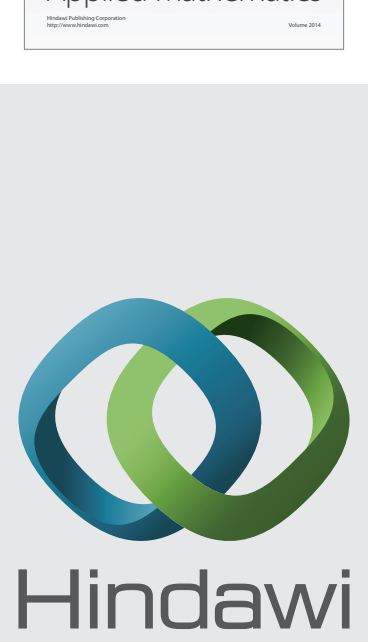

Submit your manuscripts at http://www.hindawi.com
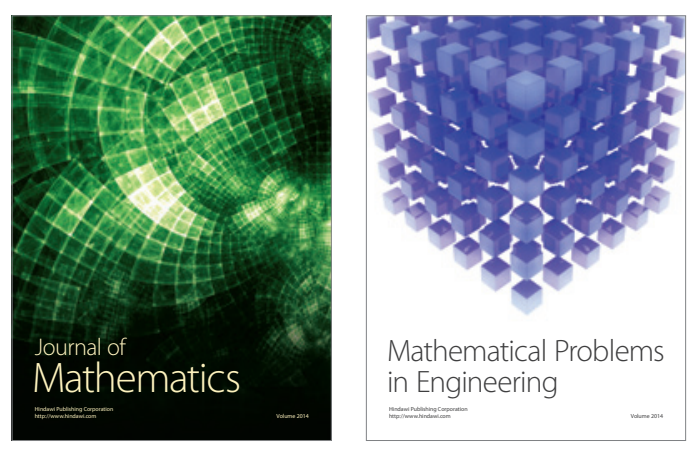

Mathematical Problems in Engineering
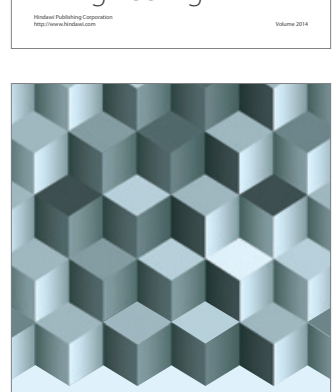

Journal of

Function Spaces
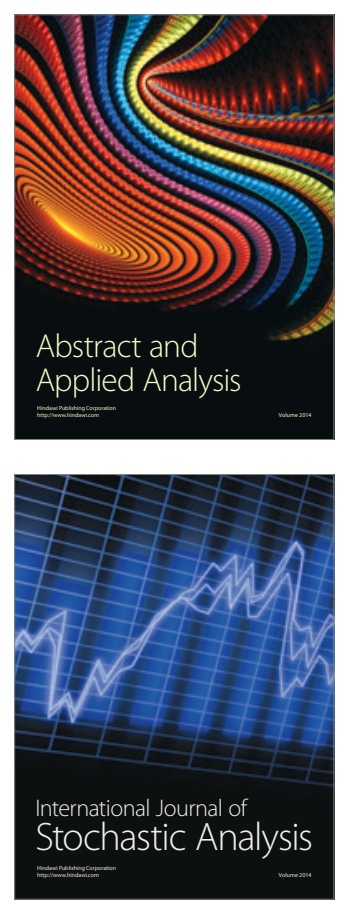

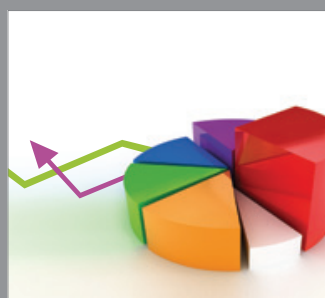

ournal of

Probability and Statistics

Promensencen
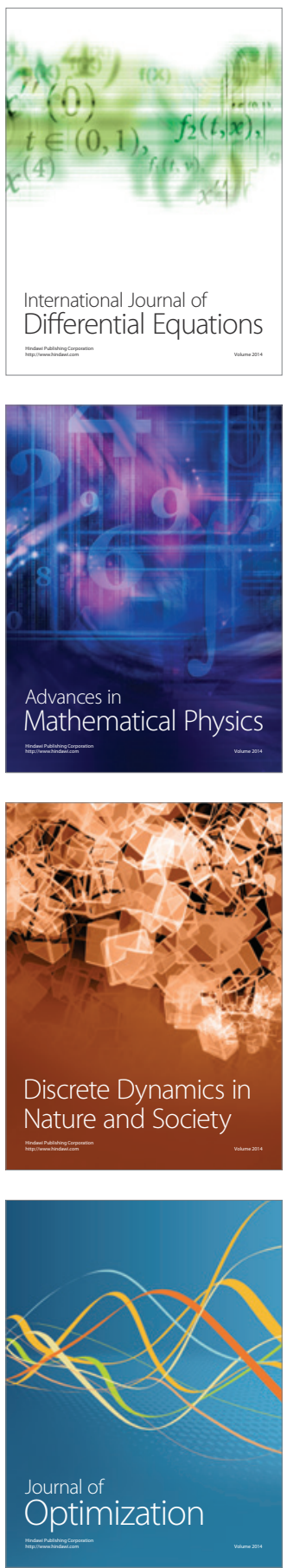